\title{
Impacto da doença crônica na qualidade de vida de idosos da comunidade em São Paulo (SP, Brasil)
}

\author{
The impact of chronic disease on the quality of life \\ of the elderly in São Paulo (SP, Brazil)
}

\author{
Alessandro Gonçalves Campolina ${ }^{1}$ \\ Patrícia Skolaude Dini ${ }^{1}$ \\ Rozana Mesquita Ciconelli ${ }^{1}$
}

${ }^{1}$ Departamento de

Medicina, Universidade

Federal de São Paulo. Rua

Botucatu 740, Vila

Clementino. 04023-900

São Paulo SP.

alecampolina@gmail.com

\begin{abstract}
The aim of this article is to evaluate the impact of chronic disease on the quality of life of elderly people living in São Paulo (SP, Brazil). Methods: cross-sectional study of individuals over 60; application of a standardized questionnaire and the Short-Form 36 item questionnaire. Statistical analysis describes the clinical and demographic data and ANOVA was used for the correlation of the SF-36 domains with age and the number of morbidities. 353 individuals were eval uated. The mean age was 71.6 years, with $48.7 \%$ men and 51.3\% women. The women presented the worst levels of quality of life in all domains evaluated. ANOVA revealed significant variation in the quality of life in several areas as the number of morbidities increased. The most compromised domain was physical aspects $(p<0.05)$. The same analysis, applied to the average of the domains by age group, showed an inverse relation of Functional Capacity $(p<0.05)$ with age. The increase in the number of morbidities and the increasing age significantly affect several areas of quality of life in the elderly. The SF-36 appears to be a valid instrument for assessing quality of life of the elderly Brazilian population.
\end{abstract} Key words Quality of life, Elderly
Resumo O objetivo deste artigo é avaliar o impacto da doença crônica na qualidade de vida de idosos da comunidade no município de São Paulo (SP). Trata-se de um estudo transversal de indivíduos acima de 60 anos, com aplicação de um questionário clínico-demográfico padronizado e do questionário genérico de qualidade de vida ShortForm 36 items (SF-36). Foram feitas análise estatística descritiva dos dados clínicos e demográficos e análise de variância (ANOVA) para correlação das médias dos domínios do SF-36 com a idade e o número de morbidades. Avaliaram-se 353 indivíduos. A média de idade foi de 71,6 anos, com 48,7\% de homens e 51,3\% de mulheres. O sexo feminino apresentou os piores níveis de qualidade de vida em todos os domínios avaliados. A análise de variância revelou uma variação significativa da qualidade de vida, em vários domínios, conforme o aumento do número de morbidades. O dominio mais comprometido foi limitação por aspectos físicos $(p<0,05)$. A mesma análise, aplicada às médias dos domínios por faixa etária, mostrou uma relação inversa do domínio capacidade funcional $(p<0,05)$ com a idade. O aumento do número de morbidades e o aumento da idade influenciam de modo significativo vários domínios da qualidade de vida dos idosos. O SF-36 apresenta-se como um instrumento válido para a avaliação de qualidade de vida da população idosa brasileira.

Palavras-chave Qualidade de Vida, Idoso 


\section{Introdução}

O envelhecimento populacional é um fenômeno mundial, que vem ganhando um ritmo cada vez mais acelerado na população brasileira, principalmente em razão da rapidez com que declinaram as taxas de fecundidade ${ }^{1}$.

Em 2000, a proporção de indivíduos com 60 anos ou mais, no Brasil, correspondia a $8,6 \%$ da população total, em comparação com $7,3 \%$ no ano de $1991^{1}$. As projeções indicam que a população de idosos em nosso país será de mais de 26,2 milhões de indivíduos em 2020, representando quase $12,4 \%$ da população total ${ }^{2}$.

Com o crescimento demográfico da população brasileira acima de 60 anos, estabelecem-se alterações nos padrões de saúde, com a redução da morbidade e mortalidade por doenças infecciosas, paralelamente ao aumento da prevalência de doenças crônicas não transmissíveis ${ }^{3}$.

Assim, diante desta realidade epidemiológica, os indicadores de saúde tradicionais tornamse insuficientes para avaliar o nível de bem-estar de uma determinada população. Ao mesmo tempo, os sinais e sintomas clássicos do adoecer mostram-se incapazes de expressar aspectos da vida relacionados ao mundo psíquico e social dos indivíduos portadores de doenças crônicas ${ }^{4}$.

Neste contexto e sob influência do conceito de Saúde da Organização Mundial da Saúde (OMS), o construto qualidade de vida passou a ser incorporado como parâmetro importante para a avaliação dos estados de saúde a partir da década de 60. Entendido como qualidade de vida subjetiva ou qualidade de vida percebida pelas pessoas, o que de fato caracteriza o conceito de qualidade de vida são os seus aspectos de subjetividade e a multidimensionalidade. A subjetividade manifesta-se através da avaliação que o indivíduo faz do seu próprio estado de saúde, derivando de um conceito baseado em uma avaliação individual que se faz de si mesmo. Já a multidimensionalidade diz respeito ao fato de esta avaliação focar diversos domínios da natureza humana: físico, psíquico, emocional, social, econômico, espiritual e outros ${ }^{5}$.

Com base nesta abordagem conceitual, vários instrumentos têm sido elaborados com a intenção de acrescentar parâmetros subjetivos à avaliação integral dos indivíduos. A literatura científica classifica tais instrumentos como genéricos e específicos. Os instrumentos genéricos são aqueles que tentam avaliar de forma global ou genérica os aspectos mais importantes relacionados à qualidade de vida dos pacientes. Os ins- trumentos específicos são aqueles que avaliam de forma individual e específica determinados aspectos da qualidade de vida ${ }^{6}$.

Ao longo dos anos, a percepção do estado de saúde e da qualidade de vida dos pacientes, bem como o impacto tanto de sua doença quanto de seu respectivo tratamento, estão sendo amplamente reconhecidos como um tópico de pesquisa em estudos clínicos e epidemiológicos ${ }^{6}$.

Apesar de existirem ainda poucos instrumentos de qualidade de vida desenvolvidos especificamente para a população idosa ${ }^{7}$, alguns estudos têm utilizado instrumentos genéricos como o Medical Outcomes Study 36-Item Short-Form Health Survey (SF-36) em populações idosas ${ }^{8-10}$.

Dificuldades com a utilização de questionários em populações idosas têm sido descritas na literatura ${ }^{11}$, e dúvidas em relação à capacidade dos idosos em completar individualmente os instrumentos têm sido cada vez mais minoradas ${ }^{12}$. Algumas estratégias vêm sendo aplicadas para facilitar a utilização do instrumento em populações idosas, dentre elas: realizar a aplicação do instrumento através de entrevistas ${ }^{8}$, realizar pequenas modificações na estrutura do questionário $^{13,14}$ e realizar entrevistas com familiares e cuidadores dos indivíduos idosos ${ }^{11}$.

Mesmo com muitas questões ainda em aberto, o SF-36 tem se apresentado aparentemente como uma medida confiável e válida, ainda quando utilizada em idosos frágeis ${ }^{10}$.

Considerando que a literatura científica brasileira é escassa em dados que reflitam a qualidade de vida dos idosos na comunidade, buscamos com este estudo traçar um perfil desses idosos no município de São Paulo (SP). Por ser esta população frequentemente acometida por doenças crônicas, focaremos nossas análises no impacto da doença crônica na qualidade de vida.

\section{Métodos}

\section{Delineamento de estudo e amostragem}

O delineamento de estudo adotado foi o observacional, transversal. A amostra foi selecionada por demanda, no período de março a abril de 2007, durante a triagem de indivíduos para a realização de cirurgia de catarata, realizada pela Universidade Federal de São Paulo (Unifesp).

Participaram desta triagem mais de 3.000 idosos (com 60 anos ou mais), que voluntariamente desejavam realizar a avaliação de acuidade visual e rastreamento da catarata. Destes foram selecio- 
nados 360, que concordaram em participar do estudo voluntariamente, após preenchimento de termo de consentimento livre e esclarecido.

Foram excluídos pacientes com doenças psiquiátricas ou comprometimentos cognitivos diagnosticados e/ou na vigência de tratamento, conforme relato dos indivíduos avaliados, pelo fato de estas condições implicarem dificuldades para compreensão dos instrumentos de pesquisa.

O presente estudo foi aprovado pelo Comitê de Ética em Pesquisa da Unifesp em 2005.

\section{Instrumentos de pesquisa}

\section{The Medical Outcomes Study}

36-Item Short-Form Health Survey (SF36)

O SF-36 tem sido amplamente utilizado por ser um questionário genérico de fácil administração e compreensão. Relativamente curto, habitualmente demanda um tempo de aplicação entre 5 e 10 minutos ${ }^{15}$.

O instrumento foi derivado inicialmente de um questionário de avaliação de saúde formado por 149 itens, desenvolvido e testado em mais de 22.000 pacientes, como parte de um estudo de avaliação de saúde, The Medical Outcomes Study (MOS).

A versão final do questionário é formada por 36 itens englobados em 8 escalas ou domínios: capacidade funcional (10 itens), aspectos físicos (4 itens), dor (2 itens), estado geral de saúde (5 itens), vitalidade (4 itens), aspectos sociais (2 itens), aspectos emocionais (3 itens), saúde mental (5 itens) e mais uma questão de avaliação comparativa entre as condições de saúde atual e a de um ano atrás. $\mathrm{O}$ instrumento avalia tanto os aspectos positivos da saúde (bem-estar) quanto os aspectos negativos (doença).

A pontuação para cada um dos 8 domínios varia de 0 (pior estado de saúde) a 100 (melhor estado de saúde). Pode-se ainda obter um escore normalizado em relação à população norte-americana para cada um dos domínios, com média de 50 e desvio padrão de 10, a partir do qual é possível calcular uma medida sumária para o componente físico e uma para o componente mental, avaliados pelo instrumento.

O SF-36 foi aplicado através de entrevistas, sendo o seu escore obtido conforme algoritmo descrito no manual publicado pelos autores ${ }^{15}$.

\section{Questionário clínico e demográfico}

Para a coleta de dados clínicos e demográficos foi utilizado um questionário estruturado dividido em duas partes, aplicado diretamente aos pacientes através de entrevistas orais.
A primeira parte compreendia itens relativos à identificação, como idade e sexo. A segunda parte do questionário compreendia itens relativos à presença de morbidades conhecidas pelos entrevistados e sobre a presença de algumas morbidades mais prevalentes na população idosa, como: diabetes mellitus (DM), hipertensão arterial sistêmica (HAS), acidente vascular cerebral (AVC), doença pulmonar obstrutiva crônica (DPOC), insuficiência cardíaca congestiva (ICC) e outras.

Os questionários eram aplicados e, após o término da entrevista, os pacientes eram encaminhados às consultas para a triagem de catarata.

\section{Análise estatística}

Os dados foram analisados através do software Statistical Package for the Social Sciences (SPSS) versão 12.0. Foi realizada a análise estatística descritiva dos dados demográficos e clínicos da população, utilizando as médias e o desvio padrão para as variáveis contínuas, e porcentagens para as variáveis categóricas. Para a comparação dos domínios do SF-36 conforme faixa etária e número de morbidades, foi empregada a análise de variância (ANOVA one-way). Adotado o intervalo de confiança de $95 \%$, os valores foram considerados significativos quando $\mathrm{p} \leq 0,05$.

\section{Resultados}

Do total de 360 indivíduos que concordaram em participar do estudo, 353 idosos (98,0\%) conseguiram completar a entrevista para a construção do perfil da qualidade de vida. O tempo médio de cada entrevista foi de 20,4 minutos.

As características demográficas e clínicas da população do estudo estão descritas na Tabela 1 . A idade média da amostra foi de 71,62 anos $(\mathrm{DP}=1,41)$. Dos avaliados, 172 pertenciam ao sexo masculino e 181 ao sexo feminino. A maior parte dos indivíduos $(65,1 \%)$ apresentava pelo menos uma morbidade.

Observamos na Tabela 2 que a maioria dos valores médios para cada domínio do questionário SF-36 situou-se entre 55 e 65 (escala de 0 a 100), e que os valores médios menores foram obtidos para os componentes: limitações por aspectos físicos e limitações por aspectos emocionais. A Tabela 2 também revela que todos os domínios foram mais comprometidos no sexo feminino.

A maior parte dos indivíduos referiu, no geral, um estado de saúde quase igual $(49,0 \%)$ ou 
um pouco pior $(20,7 \%)$ em relação ao ano anterior. Quase 50\% dos homens referiram um estado de saúde muito ou um pouco melhor em relação ao ano anterior, número que não chegou a $15 \%$ no grupo das mulheres.

$\mathrm{Na}$ Tabela 3, apresentamos a variação das médias dos domínios do SF-36 conforme o nú-

Tabela 1. Características sociodemográficas e clínicas de idosos da comunidade de São Paulo, Brasil, 1997.

\begin{tabular}{lrrr}
\hline & Total & Masculino & \multicolumn{1}{c}{ Feminino } \\
\hline Total & $353(100 \%)$ & $172(48,7 \%)$ & $181(51,3 \%)$ \\
Faixa etária & & & \\
$\quad 60-70$ & $157(44,5 \%)$ & $84(48,8 \%)$ & $73(40,3 \%)$ \\
$71-80$ & $157(44,5 \%)$ & $70(40,7 \%)$ & $87(48,1 \%)$ \\
$>80$ & $39(11 \%)$ & $18(10,5 \%)$ & $21(11,6 \%)$ \\
Morbidade & & & \\
D M & $76(21,5 \%)$ & $39(22,7 \%)$ & $37(20,4 \%)$ \\
HAS & $144(40,8 \%)$ & $54(31,4 \%)$ & $73(40,3 \%)$ \\
AVC & $19(5,4 \%)$ & $10(5,8 \%)$ & $9(5,0 \%)$ \\
DPOC & $39(11 \%)$ & $20(11,6 \%)$ & $19(10,5 \%)$ \\
ICC & $43(12,2 \%)$ & $15(8,7 \%)$ & $28(15,5 \%)$ \\
Outras & $32(9,1 \%)$ & $34(19,8)$ & $15(8,3 \%)$ \\
No morbidades & & & \\
0 & $123(34,8 \%)$ & $69(40,1 \%)$ & $54(29,8 \%)$ \\
1 & $160(45,3 \%)$ & $76(44,2 \%)$ & $84(46,4 \%)$ \\
2 & $52(14,7 \%)$ & $20(11,6 \%)$ & $32(17,7 \%)$ \\
3 ou mais & $18(5,1 \%)$ & $7(4,1 \%)$ & $11(6,1 \%)$ \\
\hline
\end{tabular}

$\mathrm{DM}=$ diabetes mellitus; $\mathrm{HAS}=$ hipertensão arterial sistêmica; $\mathrm{AVC}=$ acidente vascular cerebral; DPOC = doença pulmonar obstrutiva crônica; ICC = insuficiência cardíaca congestiva.

Tabela 2. Média dos domínios do SF-36 de idosos da comunidade de São Paulo, Brasil, 1997.

\begin{tabular}{lrll}
\hline & $\begin{array}{c}\text { Total } \\
\text { Média (DP) }\end{array}$ & $\begin{array}{l}\text { Masculino } \\
\text { Média (DP) }\end{array}$ & $\begin{array}{c}\text { Feminino } \\
\text { Média (DP) }\end{array}$ \\
\hline CFUNC & $58,48(10,60)$ & $65,20(29,78)$ & $52,09(29,65)$ \\
LAF & $54,4(17,70)$ & $56,39(39,02)$ & $52,62(41,20)$ \\
Dor & $59,6(16,30)$ & $63,05(28,89)$ & $56,27(29,02)$ \\
EGS & $61,6(21,20)$ & $64,47(21,43)$ & $59,74(23,96)$ \\
VIT & $61,0(28,00)$ & $64,98(26,42)$ & $56,29(24,41)$ \\
ASOC & $70,21(35,35)$ & $73,34(26,98)$ & $67,22(29,66)$ \\
LAE & $56,4(70,70)$ & $58,50(42,52)$ & $54,48(43,06)$ \\
SMENT & $66,49(28,28)$ & $70,86(24,65)$ & $62,34(24,48)$ \\
\hline
\end{tabular}

CFUNC = capacidade funcional; LAF = limitação por aspectos físicos; EGS = estado geral de saúde; VIT = vitalidade; ASOC = limitação por aspectos sociais; LAE = limitação por aspectos emocionais; SMENT = saúde mental. mero de morbidades. Podemos observar que a análise de variância revelou uma diferença significativa entre os grupos na maior parte dos domínios. Os domínios que apresentaram piora progressiva, conforme o aumento do número de morbidades, foram: limitações por aspectos físicos e estado geral de saúde. O domínio limitações por aspectos emocionais não apresentou variações significativas conforme o número de morbidades.

Já em relação à distribuição da média dos domínios do SF-36 por faixa etária, foi encontrada uma variação significativa apenas nos domínios capacidade funcional $(p=0,002)$ e dor $(\mathrm{p}=0,013)$. Também observamos que o domínio capacidade funcional foi o único que apresentou um declínio progressivo conforme o aumento da faixa etária.

\section{Discussão}

A utilização de questionários de qualidade de vida em idosos tem possibilitado o acesso a informações fundamentais para a atenção à saúde, apesar das dificuldades de compreensão dos instrumentos de avaliação por parte desta população ${ }^{13,16-19}$.

Em populações idosas, as taxas de itens não respondidos são altas quando o questionário é autoadministrado, podendo afetar os escores dos domínios ${ }^{17}$. Entretanto, mesmo sendo as taxas de completude afetadas em idades mais avançadas, a validade e a consistência interna do instrumento não é comprometida ${ }^{13,16,18}$.

Em um estudo realizado no Reino Unido, em que a aplicação do instrumento foi realizada por entrevista, 98,8\% dos indivíduos idosos avaliados conseguiram completar o questionário ${ }^{19}$. Estes resultados aproximam-se dos obtidos no presente estudo, em que 98,0\% dos indivíduos conseguiram completar o SF-36. A influência da escolaridade também é um aspecto a ser considerado em populações com baixo nível socioeconômico, como a avaliada neste estudo. Entretanto, optamos por não avaliar este item, visto que o SF-36 seria aplicado por entrevista.

Quando comparamos os valores médios dos domínios do SF-36 da população idosa brasileira da comunidade de São Paulo com outras populações idosas, notamos que não existe uma variação uniforme dos escores dos diversos domínios ${ }^{13,15,20}$. Entretanto, destacamos que, de maneira geral, as menores médias são encontradas para limitações por aspectos físicos, enquanto as maiores para limitações por aspectos so- 
Tabela 3. Média dos domínios do SF-36 por número de morbidades de idosos da comunidade de São Paulo, Brasil, 1997.

\begin{tabular}{lccccc}
\hline & $\mathbf{0}$ & $\mathbf{1}$ & $\mathbf{2}$ & $\mathbf{3} \mathbf{\text { ou mais }}$ \\
$\mathbf{n}=\mathbf{1 2 3}$ & $\mathbf{n}=\mathbf{1 6 0}$ & $\mathbf{n}=\mathbf{5 2}$ & $\begin{array}{c}\mathbf{p}^{*} \\
\text { (entre os grupos) }\end{array}$ \\
\hline CFUNC & $66,62(30,13)$ & $56,68(30,16)$ & $48,36(29,69)$ & $48,05(22,63)$ & $\mathbf{0 , 0 0 1}$ \\
LAF & $63,00(38,59)$ & $51,40(40,16)$ & $47,59(40,90)$ & $43,05(40,94)$ & $\mathbf{0 , 0 2 3}$ \\
Dor & $68,52(27,45)$ & $56,55(27,66)$ & $48,48(31,81)$ & $57,38(30,00)$ & $\mathbf{0 , 0 0 0}$ \\
EGS & $67,25(21,69)$ & $60,58(22,10)$ & $54,19(24,57)$ & $52,66(22,87)$ & $\mathbf{0 , 0 0 1}$ \\
VIT & $67,23(24,60)$ & $59,01(25,03)$ & $51,53(26,43)$ & $54,16(28,08)$ & $\mathbf{0 , 0 0 1}$ \\
ASOC & $73,74(27,98)$ & $70,71(26,33)$ & $61,00(32,73)$ & $68,16(34,05)$ & $\mathbf{0 , 0 5 8}$ \\
LAE & $62,31(43,33)$ & $53,72(41,50)$ & $51,90(46,42)$ & $53,61(38,24)$ & $\mathbf{0 , 3 0 5}$ \\
SMENT & $72,71(21,81)$ & $64,40(25,60)$ & $59,69(28,11)$ & $62,22(22,04)$ & $\mathbf{0 , 0 0 4}$ \\
\hline
\end{tabular}

ANOVA.

CFUNC = capacidade funcional; $\mathrm{LAF}=$ limitação por aspectos físicos; EGS = estado geral de saúde; VIT = vitalidade; ASOC = limitação por aspectos sociais; LAE = limitação por aspectos emocionais; SMENT = saúde mental.

ciais. A comparação das médias dos domínios dos idosos da comunidade de São Paulo com as médias de octagenários do Sul do Brasil ${ }^{20}$, as médias de idosos da comunidade da Inglaterra ${ }^{13}$ e a de idosos da população americana ${ }^{15}$ revela um comprometimento similar dos diferentes domínios do SF-36, reforçando sua aplicabilidade, no contexto brasileiro, para a avaliação de idosos da comunidade.

O presente estudo revela também maior comprometimento de qualidade de vida dos indivíduos idosos do sexo feminino em todos os domínios. Esses resultados corroboram o observado em outro estudo realizado em nosso meio, em que a presença de doença crônica, associada ao sexo feminino, foi o mais forte determinante de uma autopercepção ruim de saúde entre os ido$\operatorname{sos}^{2}$. As hipóteses explicativas para tais achados estão relacionadas primeiramente aos novos papéis assumidos pelas mulheres na sociedade e na família, que podem influenciar na forma como elas percebem a saúde ${ }^{21}$. Em segundo lugar, a contribuição, diferenciada por sexo, de diversas doenças crônicas e da capacidade funcional pode ser responsável por diferentes níveis de comprometimento de qualidade de vida ${ }^{2,22}$.

Os resultados do estudo demonstram ainda que os domínios que variaram conforme a faixa etária, a capacidade funcional e a dor. Destacamos que o domínio capacidade funcional foi o único que declinou progressivamente com o aumento da faixa etária. Apesar de esses resultados poderem sofrer a influência do tamanho amostral em cada uma das faixas etárias, achamos que a capacidade funcional é um aspecto central da qualidade de vida do idoso, que sofre influências com o aumento da idade, conforme já demonstrado em outros estudos ${ }^{23,24,25}$.

Nesse sentido, a capacidade funcional é um domínio que demonstra variação conforme a faixa etária, revelando-se um alvo de investimento importante na avaliação e na promoção da saúde de indivíduos idosos. Resultados do estudo Epidoso, que acompanhou uma coorte de idosos por mais de 10 anos em São Paulo, reforçam a importância da capacidade funcional, que, junto com o estado cognitivo, foi um dos únicos fatores de risco para mortalidade que são mutáveis e se mantiveram independentes após análise multivariada ${ }^{24}$.

A análise de variância das médias dos domínios do SF-36 por número de comorbidades revelou-se significativa para vários domínios. Os dados demonstram que o aumento do número de doenças crônicas impacta várias domínios da qualidade de vida. O SF-36 apresenta-se, assim, como um bom perfil capaz de apresentar os domínios mais comprometidos e que sofrem maiores variações com a idade. Em nossa amostra, destacamos que o único domínio que não sofreu variação com o número de morbidades foi a limitação por aspectos emocionais, que acreditamos ser em parte explicado pela exclusão de indivíduos com comprometimento cognitivo. Também destacamos o maior comprometimento de qualidade de vida, relacionado a limitações por aspectos físicos, escala esta que tem sido mais sensível ao impacto da doença crônica em outros estudos utilizando o SF-36, e que merece atenção especial na avaliação do idoso ${ }^{15}$. 
Como limitações principais do presente estudo, destacamos que a amostragem por demanda de serviço, em um centro de referência, pode não refletir rigorosamente todo o universo de idosos residentes na comunidade; a utilização de um instrumento genérico de qualidade de vida pode não avaliar aspectos mais específicos da qualidade de vida na terceira idade, e a avaliação autorreferida de morbidades pode não refletir de modo fidedigno os diagnósticos dos indivíduos avaliados.

\section{Conclusões}

O bem-estar na velhice está relacionado com o equilíbrio entre várias dimensões da qualidade de vida. O aumento do número de morbidades e o aumento da idade influenciam de modo significativo vários domínios da qualidade de vida dos indivíduos idosos, em especial a capacidade funcional, que parece ser o único domínio a sofrer influencia diretamente da idade.

O SF-36 tem se apresentado como instrumento potencialmente válido para avaliação de qualidade de vida na população idosa brasileira, mostrando-se uma alternativa a ser incorporada em protocolos de atenção à saúde do idoso.

\section{Colaboradores}

AG Campolina, PS Dini e RM Ciconelli participaram igualmente de todas as etapas de elaboração do artigo. 


\section{Referências}

1. Carvalho JAM, Garcia RA. O envelhecimento da população brasileira: um enfoque demográfico. Cad Saude Publica 2003; 19(3):725-733.

2. Rodrigues RN, Alves LC. Determinantes da autopercepção de saúde entre idosos do município de São Paulo, Brasil. Rev Panam Salud Publica 2005; 17(5/6): 333-341.

3. Chaimowicks F. A saúde dos idosos brasileiros às vésperas do século XXI: problemas, projeções e alternativas. Rev Saude Publica 1997; 31(2):184-200.

4. Ebrahim S. Clinical and Public Health perspectives and applications of health related quality of life measurement. Soc Sci Med 1995; 41(10):1383-1394.

5. The WHOQOL Group. The World Health Organization Quality of Life Assessment (WHOQOL): position paper from the World Health Organization. Soc Sci Med 1995; 41(10):1403-1409.

6. Guyatt GH, Feeny DH, Patrick DL. Measuring health related quality of life. Ann Intern Med 1993; 118(8):622-629.

7. Paschoal SMP. Qualidade de vida na velhice. In: Freitas EV, Py L, Néri AL, Cançado HAX, Gorzoni ML, Rocha SM, organizadores. Tratado de geriatria e gerontologia. Rio de Janeiro: Guanabara Koogan; 2002. p. 79-84.

8. Mahony PG, Rodgers H, Thomson RG, Dobson R, James OFW. Is the SF-36 suitable for assessing health status of older stroke patients? Age and Ageing 1998; 27(1):19-22.

9. Mozes B, Maor Y, Shmueli A. The competing effects of disease states on quality of life of the elderly. Quality o Life Research 1999; 8(1/2):93-99.

10. Stadnyk K, Calder J, Rockwood K. Testing the measurement properties of the Short Form-36 Health Survey in a frail elderly population. J Clin Epidemiol 1998; 51(10):827-835.

11. Ball AE, Russell EM, Seymour DG, Primrose WR, Garratt AM. Problems in using health survey questionnaires in older patients with physical disabilities. Gerontology 2001; 47(6):334-340.

12. Dempster M, Donnelly M. How well do elderly people complete individualized quality of life measures: an exploratory study. Quality of Life Research 2000; 9(4):369-375

13. Walters SJ, Munro JF, Brazier JE. Using the SF-36 with older adults: a cross-sectional communitybased survey. Age and Ageing 2001; 30(4):337-343.

14. Wolinsky FD, Stump TE. A measurement model of the medical outcomes Study 36-Item Short-Form Health Survey in a clinical sample of disadvantaged, older, black and white men and women. Med Care 1996; 34(6):537-548.

15. Ware JE, Snow KK, Kosinski M, Gandek B. SF-36 Health Survey: manual and interpretation guide. Boston: New England Medical Center; 1993.
16. Hayes V, Morris J, Wolfe C, Morgan M. The SF-36 Health Survey Questionnaire: is it suitable for use with older adults? Age and Ageing 1995; 24(2):120125.

17. Mallison S. The Short-Form 36 and older people: some problems encountered when using postal administration. J Epidemiol Community Health 1998; 52(5):324-328.

18. Chia EM, Chia EM, Rochtchina E, Wang JJ, Mitchell P. Utility and validity of the Self-administered SF-36: findings from an older population. Ann Acad Med Singapore 2006; 35(7):461-467.

19. Lyons RA, Perry HM, Littlepage NC. Evidence for the validity of Short-Form 36 Questionnaire (SF36 ) in an elderly population. Age and Ageing 1994; 23(3):182-184.

20. Xavier FMF, Ferraz MPT, Bertollucci P, Poyares D, Moriguchi EH. Episódio depressivo maior, prevalência e impacto sobre qualidade de vida, sono e cognição em octagenários. Rev Bras Psiquiatr 2001; 23(2):62-70

21. Lorenzi DRS, Baracat EC, Saciloto B, Padilha Jr. I. Fatores associados à qualidade de vida após menopausa. Rev Assoc Med Bras 2006; 52(5):312-317.

22. Camargos MCS, Perpétuo IHO, Machado CJ. Expectativa de vida com incapacidade funcional em idosos em São Paulo, Brasil. Rev Panam Salud Publica 2005; 17(5/6):379-386.

23. Alves LC, Leimann BCQ, Vasconcelos MEL, Carvalho MS, Vasconcelos AGG, Fonseca TCO, Lebrão ML, Laurenti R. A influência das doenças crônicas na capacidade funcional dos idosos do Município de São Paulo, Brasil. Cad Saude Publica 2007; 23(8):1924-1930.

24. Ramos LR. Epidemiologia do envelhecimento. In: Freitas EV, Py L, Néri AL, Cançado HAX, Gorzoni ML, Rocha SMl, organizadores. Tratado de geriatria e gerontologia. Rio de Janeiro: Guanabara Koogan; 2002. p. 72-78.

25. Barbosa AR, Souza JMP, Lebrão ML, Laurenti R, Marucci MFN. Functional limitations of Brazilian elderly by age and gender differences: data from SABE Survey. Cad Saude Publica 2005; 21(4):11771185.

Artigo apresentado em 29/08/2008

Aprovado em 09/02/2009

Versão final apresentada em 18/2/2009 